Sonderdruck aus

\title{
Archiv der Pharmazie
}

317. Band, Heft 5, Seite $438-442$

Verlag Chemie GmbH, Weinheim (Bergstr.)

Cleavage of 1-Benzyltetrahydroisoquinolines to Secondary Amines via Urethanes

Wan-Joo Kimª), Dong-Ung Leea) and Wolfgang Wiegrebe ${ }^{\left.\mathrm{b})^{*}+\right)}$ 
Arch. Pharm. (Weinheim) 317, 438-442 (1984)

\title{
Cleavage of 1-Benzyltetrahydroisoquinolines to Secondary Amines via Urethanes
}

\author{
Wan-Joo Kim ${ }^{\text {a)}}$, Dong-Ung Lee ${ }^{\text {a) }}$ and Wolfgang Wiegrebe ${ }^{\text {b)*)+) }}$
}

a) Korea Advanced Institute of Science and Technology, P.O. Box 131, Cheongryang, Seoul (Korea) and

b) Institute of Pharmacy, University of Regensburg, P.O. Box 397, D 8400 Regensburg

(Germany)

Eingegangen am 7. März 1983

2,2,2-Trichloroethyl chloroformate and benzyl chloroformate cleave 1-benzyl-1,2,3,4-tetrahydro-2-methylisoquinolines of type 1 to yield tertiary stilbene urethanes of type 2 which are easily reduced or hydrogenolyzed to secondary amines. $6^{\prime}$-(Hydroxymethyl)-laudanosine (4) is converted by these reagents to the isochroman urethanes 11 and 12 which are split to yield the secondary amine 8 without the isochroman moiety being attacked.

\section{Spaltung von 1-Benzyl-tetrahydroisochinolinen über Urethane zu sekundären Aminen}

Chlorameisensäure-2,2,2-trichlorethylester bzw. Chlorameisensäurebenzylester spalten 1-Benzyl-1,2,3,4-tetrahydro-2-methylisochinoline vom Typ 1 zu tertiären Stilben-Urethanen vom Typ 2, die unter milden Bedingungen reduktiv bzw. hydrogenolytisch zu sekundären Aminen gespalten werden. 6'-Hydroxymethyllaudanosin (4) reagiert mit diesen Reagentien zu den Isochroman-Urethanen 11 bzw. 12, die ohne Angriff am Isochromanring zum sekundären Amin 8 umgesetzt werden.

Gadamer and $\mathrm{Knoch}^{1)}$ have found that ethyl chloroformate transforms 1-benzyl-1,2,3,4-tetrahydro-2-methylisoquinolines (e.g. 1) easily into stilbene-urethanes (e. g. 2). Unfortunately the CO-OEt-moiety is fixed very strongly to the $\mathrm{N}$-atom ${ }^{1,2)}$, so that the synthetic value of this reagent is diminished. $v$. Bruchhausen and $\mathrm{Knabe}^{3)}$ have overcome this disadvantage by $\mathrm{LiAlH}_{4}$-reduction of the urethane to the $\mathrm{N}, \mathrm{N}$-dimethyl function ${ }^{3)}$ (e. g. 3). Moreover ethyl chloroformate degradation of 6 '-hydroxymethyllaudanosine (4) leads to the 3-phenylisochroman ring system 5 , and the $\mathrm{LiAlH}_{4}$ reduction of the $\mathrm{N}$-methylcarbamate function in 5 was easily achieved to produce the $\mathrm{N}, \mathrm{N}$-dimethylamine derivative $6^{4}$.

${ }^{+}$) Herrn Professor Brockmann, Göttingen, zum 80. Geburtstag freundlichst gewidmet.

$0365-6233 / 84 / 0505-0438 \$ 02.50 / 0$

○ Verlag Chemie GmbH, Weinheim 1984 
The corresponding $\mathrm{N}$-monomethylamines 7 and 8 can serve as versatile intermediates in alkaloid synthesis. The syntheses of 7 and 8 from the carbamates 2 and 5 have not yet been reported. Hydrolyses of carbamates with strong acids ${ }^{5)}$ or strong bases ${ }^{6}$ are commonly known, but the isochroman ring in $\mathbf{5}$ may not be compatible with these hydrolytic conditions. So it seemed worthwhile to obtain urethanes which can be split under mild conditions: 2,2,2-trichloroethyl chloroformate (TEC) and benzyl chloroformate (BC) enabled us to prepare the secondary amines 7 and 8 . The trichloroethoxycarbonyl moiety $^{7,8)}$ is well known as a protecting group of alcohols and amines in alkaloids and steroids. It is particulary useful for compounds containing structures sensitive to acidic or basic conditions on account of its facile removal by zinc/acetic acid reduction. The reaction of laudanosine (1) with TEC affords the 2,2,2-trichloroethyl-N-methylcarbamate 9. Upon reduction of 9 with zinc dust in glacial acetic acid at room temp., the $\mathrm{N}$-methylamine 7 was obtained. The reaction of $6^{\prime}$-hydroxymethyllaudanosine (4) with TEC produced the corresponding carbamate $\mathbf{1 1}$, which was reduced to the $\mathrm{N}$-methylamine 8 .

The benzyloxycarbonyl group is also widely used as protecting group in organic syntheses $^{9-18)}$, the removal of this function can be achieved by Pd-catalyzed hydrogenolysis. When laudanosine (1) and 6'-hydroxymethyllaudanosine (4) were reacted with BC, the corresponding benzyl carbamates 10 and 12 were formed. While the hydrogenolysis of the benzylcarbamate 12 yielded the secondary amine 8, the benzylcarbamte 10 was transformed into dihydro-7 $=\mathbf{1 3}$ by concomitant hydrogenation of the stilbene double bond. 7 and 8 were reacted with ethyl chloroformate to the $\mathrm{N}$-methylcarbamates 2 and 5 , so confirming the structure assignments.
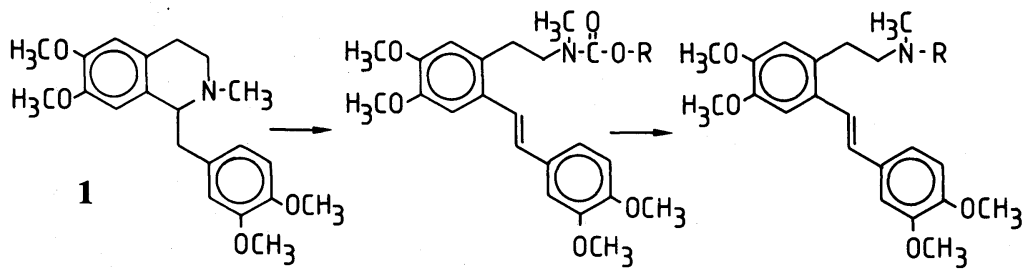
2: $\mathrm{R}=\mathrm{C}_{2} \mathrm{H}_{5}$
3: $\mathrm{R}=\mathrm{CH}_{3}$
9: $\mathrm{R}=\mathrm{CH}_{2}-\mathrm{CCl}_{3}$
7: $\mathrm{R}=\mathrm{H}$
10: $\mathrm{R}=\mathrm{CH}_{2}-\emptyset$
13: dihydro-7

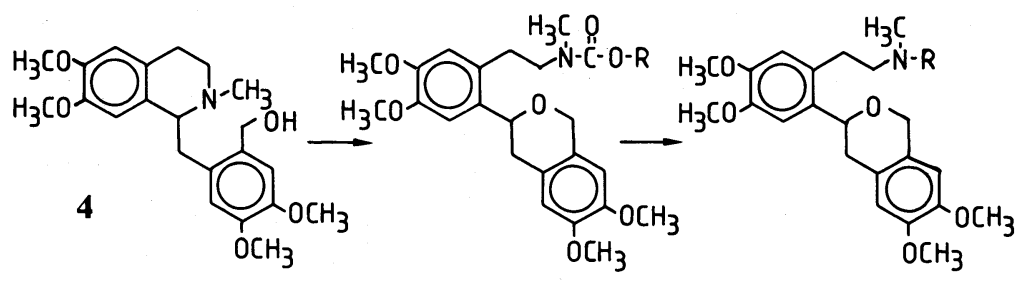
5: $\mathrm{R}=\mathrm{C}_{2} \mathrm{H}_{5}$
6: $\mathrm{R}=\mathrm{CH}_{3}$
11: $\mathrm{R}=\mathrm{CH}_{2}-\mathrm{CCl}_{3}$
8: $R=H$
12: $\mathrm{R}=\mathrm{CH}_{2}-\emptyset$ 


\section{Experimental Part}

$M P$ : Thomas-Hoover melting point apparatus, uncorr. $-{ }^{1} H$-NMR spectra: Varian EM 360 A (60 $\mathrm{MHz}$ ) in $\mathrm{CDCl}_{3}$, TMS as int. stand. - IR spectra: Perkin-Elmer $735 \mathrm{~B}$ spectrophotometer, $\mathrm{KBr}$ pellets. - UV-spectra: Uvikon 810 spectrophotometer (Kontron). - Elementary Analyses: Perkin-Elmer 240 $\mathrm{C}, \mathrm{H}, \mathrm{N}$-analyzer. - All compound were checked by tlc (chloroform-acetone-methanol $2: 2: 1$, v/v), using Baker-flex flexible sheets $7.5 \times 2.5 \mathrm{~cm}$. - The UV-spectra correspond to those of similar compounds: isochromans: ${ }^{4)}$, stilbenes: ${ }^{3)}{ }^{\bullet}$

\section{Isochromans}

$6^{\prime}$-Hydroxymethyllaudanosine (4) was prepared as reported ${ }^{19,20)}$.

\section{$3-\left[2^{\prime}-(\beta-N-2,2,2-T r i c h l o r o e t h o x y c a r b o n y l-N-m e t h y l-a m i n o e t h y l)-4^{\prime}, 5^{\prime}\right.$-dimethoxyphenyl]-6,7- dimethoxyisochroman (11)}

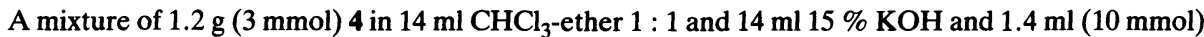
2,2,2-trichloroethyl chloroformate (TEC) was reacted under reflux for $2 \mathrm{~h}$. Another $14 \mathrm{ml} 15 \% \mathrm{KOH}$ and $1.4 \mathrm{ml}$ TEC were added and $2 \mathrm{~h}$ later, the excess of TEC was destroyed by heating with $7 \mathrm{ml} 15 \%$ $\mathrm{KOH}$. After $1 \mathrm{~h}$ the org. layer was separated and concentrated to give a yellow oily residue which was crystallized with ether: $1.4 \mathrm{~g}(85 \%)$, mp. $142^{\circ}, \mathrm{Rf}=0.79 . \mathrm{C}_{25} \mathrm{H}_{30} \mathrm{Cl}_{3} \mathrm{NO}_{7}(562.7)$ Calcd. C 53.3 H 5.33 N 2.5 Found C 53.7 H 5.22 N 2.4. IR: $1715 \mathrm{~cm}^{-1}(\mathrm{CO}) .{ }^{1} \mathrm{H}-\mathrm{NMR}: \delta(\mathrm{ppm})=2.70-4.06(\mathrm{~m} ; 6 \mathrm{H}$, $\left.-\mathrm{CH}_{2}-\right), 2.93\left(\mathrm{~s} ; 3 \mathrm{H},-\mathrm{NCH}_{3}\right), 3.87\left(\mathrm{~s} ; 12 \mathrm{H},-\mathrm{OCH}_{3}\right), 4.68(\mathrm{t} ; \mathrm{J}=5 \mathrm{~Hz}, 1 \mathrm{H},-\mathrm{O}-\mathrm{CH}-), 4.70(\mathrm{~s} ; 2 \mathrm{H}$, $\left.-\mathrm{CH}_{2}-\mathrm{CCl}_{3}\right), 4.93\left(\mathrm{~s} ; 2 \mathrm{H},-\mathrm{O}-\mathrm{CH}_{2}-\right), 6.56,6.61,6.70$ and $7.03(4 \times \mathrm{s} ; 4 \mathrm{H}$, aromatic $\mathrm{Hs})$.

\section{3-[2'-( $\beta$-N-Benzyloxycarbonyl-N-methyl-aminoethyl)-4', $5^{\prime}$-dimethoxyphenyl]-6,7-dimethoxyiso- chroman (12)}

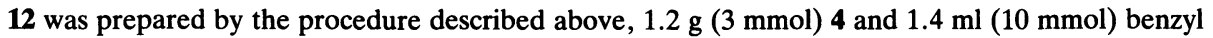
chloroformate (BC) were used: $1.4 \mathrm{~g}(91 \%)$, mp. $143^{\circ}$ from ether, $\mathrm{Rf}=0.77 . \mathrm{C}_{30} \mathrm{H}_{35} \mathrm{NO}_{7}(521.5)$ Calcd. C 69.1 H 6.71 N 2.7 Found C $68.6 \mathrm{H} 6.65 \mathrm{~N}$ 2.6. IR: $1710 \mathrm{~cm}^{-1}(\mathrm{CO}) .{ }^{1} \mathrm{H}-\mathrm{NMR}: \delta(\mathrm{ppm})=$ $2.67-4.00\left(\mathrm{~m} ; 6 \mathrm{H},-\mathrm{CH}_{2}-\right), 2.87\left(\mathrm{~s} ; 3 \mathrm{H},-\mathrm{NCH}_{3}\right), 3.80\left(\mathrm{~s} ; 3 \mathrm{H},-\mathrm{OCH}_{3}\right), 3.86\left(\mathrm{~s} ; 9 \mathrm{H},-\mathrm{OCH}_{3}\right), 4.80(\mathrm{t} ; \mathrm{J}$ $=5 \mathrm{~Hz}, 1 \mathrm{H},-\mathrm{O}-\mathrm{CH}-), 4.87\left(\mathrm{~s} ; 2 \mathrm{H},-\mathrm{O}-\mathrm{CH}_{2}-\right), 5.05\left(\mathrm{~s} ; 2 \mathrm{H},-\mathrm{CH}_{2}-\right.$ phenyl $), 6.56,6.63$ and $7.06(3 \times \mathrm{s}$; $4 \mathrm{H}$, aromatic $\mathrm{Hs}), 7.30\left(\mathrm{~s} ; 5 \mathrm{H},-\mathrm{C}_{6} \mathrm{H}_{5}\right)$.

\section{3-[2'-( $\beta-N$-Methylaminoethyl)-4',5'-dimethoxyphenyl]-6,7-dimethoxyisochroman (8)}

1) A solution of $1.1 \mathrm{~g}(2 \mathrm{mmol}) 11 \mathrm{in} 5 \mathrm{ml}$ dioxan and $10 \mathrm{ml}$ glacial acetic acid and $1.0 \mathrm{~g}$ zinc dust (zinc was activated according to ${ }^{21)}$ ) was stirred for $4 \mathrm{~h}$ at room temp. After removal of zinc, the filtrate was made strongly alkaline with conc. $\mathrm{NaOH}$ and extracted with $\mathrm{CHCl}_{3}$. The organic layer was concentrated to give an oily residue, which was dissolved in ether. To this solution conc. $\mathrm{HCl}$ was added dropwise until the salt formation was completed. The solution of $8-\mathrm{HCl}$ in little water was made alkaline to give 8: $0.5 \mathrm{~g}(65 \%)$, mp. $115^{\circ}$ from ether, $\mathrm{Rf}=0.14 . \mathrm{C}_{22} \mathrm{H}_{29} \mathrm{NO}_{5}(387.5)$ Calcd. $\mathrm{C} 68.2 \mathrm{H}$ $7.48 \mathrm{~N}$ 3.6 Found C $67.8 \mathrm{H} 7.67 \mathrm{~N}$ 3.5. IR: $2750-3050 \mathrm{~cm}^{-1}(\mathrm{NH}) .{ }^{1} \mathrm{H}-\mathrm{NMR}: \delta(\mathrm{ppm})=1.79(\mathrm{~s} ; 1 \mathrm{H}$, $-\mathrm{NH}), 2.44\left(\mathrm{~s} ; 3 \mathrm{H},-\mathrm{NCH}_{3}\right), 2.60-3.23\left(\mathrm{~m} ; 6 \mathrm{H},-\mathrm{CH}_{2}-\right), 3.91\left(\mathrm{~s} ; 12 \mathrm{H},-\mathrm{OCH}_{3}\right), 4.87(\mathrm{t} ; \mathrm{J}=5 \mathrm{~Hz}, 1 \mathrm{H}$, $-\mathrm{O}-\mathrm{CH}-), 4.97\left(\mathrm{~s} ; 2 \mathrm{H},-\mathrm{O}-\mathrm{CH}_{2}-\right), 6.60,6.67,6.77$ and $7.13(4 \times \mathrm{s} ; 4 \mathrm{H}$, aromatic $\mathrm{Hs})$.

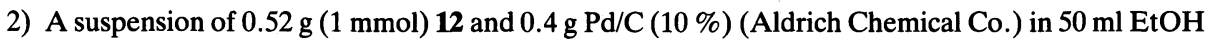
was shaken with $\mathrm{H}_{2}$ for $1 \mathrm{~h}$ at atomospheric pressure. After removal of $\mathrm{Pd} / \mathrm{C}$, the filtrate was concentrated and the residue treated with conc. $\mathrm{HCl}$ as described above. Usual work-up (see above) gave $0.24 \mathrm{~g} 8(62 \%)$. 
3-[2'-( $\beta$-N-Ethoxycarbonyl-N-methyl-aminoethyl)-4',5'-dimethoxyphenyl]-6,7-dimethoxyisochroman (5)

$0.2 \mathrm{~g}(0.5 \mathrm{mmol}) 8$ in $5 \mathrm{ml} \mathrm{CHCl}_{3}$ were added to $0.1 \mathrm{ml}$ ethyl chloroformate and treated with $4-5$ drops triethylamine. The mixture was stirred for a few min at room temp. and concentrated, usual work-up led to 5 in $95 \%$ yield, which was crystallized with ether. Its physical data correspond to those of an authentic sample $\mathrm{e}^{20)}$.

\section{Stilbenes}

\section{2-( $\beta-N-2,2,2-T r i c h l o r o e t h o x y c a r b o n y l-N-m e t h y l-a m i n o e t h y l)-4,5,3^{\prime}, 4^{\prime}$-tetramethoxystilbene (9)}

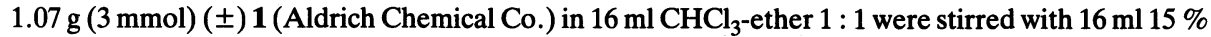
$\mathrm{KOH}$ and $1.4 \mathrm{ml}(10 \mathrm{mmol})$ TEC on the steam bath for $2 \mathrm{~h}$; the organic layer was concentrated to give a yellow oily residue which was crystallized with ether: $1.2 \mathrm{~g}(75 \%), \mathrm{mp} .135-136^{\circ}, \mathrm{Rf}=0.7$. $\mathrm{C}_{24} \mathrm{H}_{28} \mathrm{Cl}_{3} \mathrm{NO}_{6}(532.9)$ Calcd. C 54.1 H 5.25 N 2.6 Found C $54.2 \mathrm{H} 5.27 \mathrm{~N} 2.8$. IR: $1710 \mathrm{~cm}^{-1}$ (CO). ${ }^{1} \mathrm{H}-\mathrm{NMR}: \delta(\mathrm{ppm})=2.93\left(\mathrm{~s} ; 3 \mathrm{H},-\mathrm{NCH}_{3}\right), 2.56-4.13\left(\mathrm{~m} ; 4 \mathrm{H},-\mathrm{CH}_{2}-\right), 3.93\left(\mathrm{~s} ; 6 \mathrm{H},-\mathrm{OCH}_{3}\right), 3.97(\mathrm{~s}$; $\left.\left.3 \mathrm{H},-\mathrm{OCH}_{3}\right), 4.00\left(\mathrm{~s} ; 3 \mathrm{H},-\mathrm{OCH}_{3}\right), 4.67\left(\mathrm{~s} ; 2 \mathrm{H},-\mathrm{CH}_{2}-\mathrm{CCl}_{3}\right)^{*}\right), 6.57-7.43(\mathrm{~m} ; 7 \mathrm{H}$, aromatic and vinyl Hs).

\section{2-( $\beta-N$-Benzyloxycarbonyl-N-methyl-aminoethyl)-4,5,3',4'-tetramethoxystilbene (10)}

10 was prepared by the procedure and work-up described above, $1.07 \mathrm{~g}(3 \mathrm{mmol}) 1$ and $1.4 \mathrm{ml}(10$ mmol) BC were used: $1.0 \mathrm{~g}(71 \%)$, mp. 126-127 ${ }^{\circ}$ from ether, $\mathrm{Rf}=0.69 . \mathrm{C}_{29} \mathrm{H}_{33} \mathrm{NO}_{6}(491.5) \mathrm{Calcd}$. C 70.9 H 6.71 N 2.9 Found C 71.0 H 6.92 N 2.9. IR: $1680 \mathrm{~cm}^{-1}(\mathrm{CO}) .{ }^{1} \mathrm{H}-\mathrm{NMR}: \delta(\mathrm{ppm})=2.87(\mathrm{~s} ; 3 \mathrm{H}$, $\left.-\mathrm{NCH}_{3}\right), 2.57-4.10\left(\mathrm{~m} ; 4 \mathrm{H},-\mathrm{CH}_{2}-\right), 3.83\left(\mathrm{~s} ; 3 \mathrm{H},-\mathrm{OCH}_{3}\right), 3.90\left(\mathrm{~s} ; 6 \mathrm{H},-\mathrm{OCH}_{3}\right), 3.96(\mathrm{~s} ; 3 \mathrm{H}$, $\left.-\mathrm{OCH}_{3}\right), 5.07\left(\mathrm{~s} ; 2 \mathrm{H},-\mathrm{CH}_{2}\right.$-phenyl), 6.40-7.50 (m; $7 \mathrm{H}$, aromatic and vinyl $\left.\mathrm{Hs}\right), 7.33$ (s; $5 \mathrm{H}$, $\left.-\mathrm{C}_{6} \mathrm{H}_{5}\right)$.

\section{2-( $\beta$-N-Methylaminoethyl)-4,5,3', 4'-tetramethoxystilbene (7)}

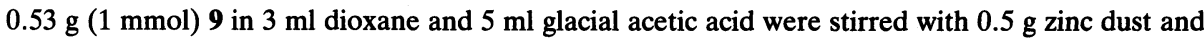
worked up as described for 11 to 8: $0.23 \mathrm{~g}(65 \%)$, mp. $106^{\circ}$ from ether, $\mathrm{Rf}=0.13 . \mathrm{C}_{21} \mathrm{H}_{27} \mathrm{NO}_{4}(357.5)$ Calcd. C 70.5 H 7.55 N 3.9 Found C 70.4 H 7.32 N 3.9. IR: $2750-3000 \mathrm{~cm}^{-1}(\mathrm{NH}) .{ }^{1} \mathrm{H}-\mathrm{NMR}$ : $\delta$ (ppm) $=2.47\left(\mathrm{~s} ; 3 \mathrm{H},-\mathrm{NCH}_{3}\right), 2.70-3.20\left(\mathrm{~m} ; 4 \mathrm{H},-\mathrm{CH}_{2}-\right), 3.00(\mathrm{~s} ; 1 \mathrm{H},-\mathrm{NH}), 3.93\left(\mathrm{~s} ; 6 \mathrm{H},-\mathrm{OCH}_{3}\right), 3.97(\mathrm{~s} ;$ $\left.6 \mathrm{H},-\mathrm{OCH}_{3}\right), 6.63-7.30(\mathrm{~m} ; 7 \mathrm{H}$, aromatic and vinyl $\mathrm{Hs})$.

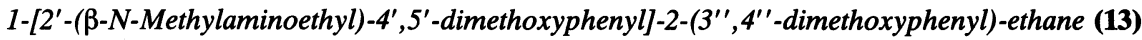

$0.49 \mathrm{~g}(1 \mathrm{mmol}) 10$ in $40 \mathrm{ml} \mathrm{CHCl}$ were hydrogenated with $0.4 \mathrm{~g} 10 \% \mathrm{Pd} / \mathrm{C}$ at room temp. under atmospheric pressure for $6 \mathrm{~h}$ (absorption $45 \mathrm{ml}$, calcd. $44.8 \mathrm{ml}$ ). After filtration the solution was concentrated i. vac., the oily residue was crystallized with ether. $0.26 \mathrm{~g}(71 \%), \mathrm{mp} .184^{\circ}, \mathbf{R f}=0.6$. 13-p-Nitrobenzoylamide was prepared according to Schotten-Baumann, mp. $125^{\circ}$ (EtOH). $\mathrm{C}_{28} \mathrm{H}_{32} \mathrm{~N}_{2} \mathrm{O}_{7}(508.6)$ Calcd. C 66.1 H 6.35 N 5.5 Found C 66.1 H 6.23 N 5.5. Data of 13: MS (70 eV): $\mathrm{m} / \mathrm{z} 359\left(\mathrm{M}^{+}, 6 \%\right), 316\left[\mathrm{M}^{+} \cdot-\left(\mathrm{CH}_{3}-\mathrm{N}=\mathrm{CH}_{2}\right), 56 \%,{ }^{*} 278.15\right], 165\left[316-\left(\cdot \mathrm{CH}_{2}-\mathrm{C}_{6} \mathrm{H}_{3}\left(\mathrm{OCH}_{3}\right)_{2}\right), 100\right.$

*) Note added in proof: The $90 \mathrm{MHz}-\mathrm{NMR}$-spectrum of 9 shows two s for $-\mathrm{CH}_{2}-\mathrm{CCl}_{3}$ at $\delta=4.62 \mathrm{ppm}$ and $4.68 \mathrm{ppm}$. Heating up to $85^{\circ}$ leads to one $s$ at $\delta=4.57 \mathrm{ppm}$, after cooling the sample shows two $s$ at the original positions again. The origin of this doubling is unknown, this phenomenon is not observed in dihydro-9. 
$\%$, *86.16], $151\left({ }^{+} \mathrm{CH}_{2}-\mathrm{C}_{6} \mathrm{H}_{3}\left(\mathrm{OCH}_{3}\right)_{2}, 31 \%\right), 44\left(\mathrm{H}_{3} \mathrm{C}-{ }^{+} \mathrm{NH}=\mathrm{CH}_{2}, 78 \%\right)$. IR: $2700-3200 \mathrm{~cm}^{-1}$ $(\mathrm{NH}) .{ }^{1} \mathrm{H}-\mathrm{NMR}: \delta(\mathrm{ppm})=2.66\left(\mathrm{~s} ; 3 \mathrm{H},-\mathrm{NCH}_{3}\right), 2.66-3.40\left(\mathrm{~m} ; 4 \mathrm{H},-\mathrm{CH}_{2}-\right), 2.90(\mathrm{~s} ; 4 \mathrm{H}$, $\left.\mathrm{Ar}-\mathrm{CH}_{2}-\mathrm{CH}_{2}-\mathrm{Ar}\right), 3.90\left(\mathrm{~s} ; 6 \mathrm{H},-\mathrm{OCH}_{3}\right), 3.93\left(\mathrm{~s} ; 3 \mathrm{H},-\mathrm{OCH}_{3}\right), 3.96\left(\mathrm{~s} ; 3 \mathrm{H},-\mathrm{OCH}_{3}\right), 6.80$ and 6.88 $(2 \times \mathrm{s}$; $5 \mathrm{H}$, aromatic Hs), 9.43 (broad; $1 \mathrm{H},-\mathrm{NH})$. UV (methanol) $\lambda \max (\log \varepsilon): 212$ (4.26), 2.30 (4.22), $280 \mathrm{~nm}(3.80)$.

\section{2-( $\beta$-N-Ethoxycarbonyl-N-methyl-aminoethyl)-4,5,3', $4^{\prime}$-tetramethoxystilbene (2)}

$0.18 \mathrm{~g}(0.5 \mathrm{mmol}) 7 \mathrm{in} 5 \mathrm{ml} \mathrm{CHCl}{ }_{3}$ were added to $0.1 \mathrm{ml}$ ethyl chloroformate and treated with a few drops of triethylamine. According to the procedure used in the synthesis of 5 from 8, 2 was obtained in $98 \%$ yield and identified by comparison of its physical data with those of an authentic sample ${ }^{1)}$.

\section{References}

1 J. Gadamer and F. Knoch, Arch. Pharm. (Weinheim) 259, 135 (1921).

2 F.v. Bruchhausen and H. Schultze, Arch. Pharm. (Weinheim) 267, 617 (1929).

3 F.v. Bruchhausen and J. Knabe, Arch. Pharm. (Weinheim) 287, 601 (1954).

4 W. Wiegrebe, Arch. Pharm. (Weinheim) 301, 672 (1968).

5 D. L. Trepanier and S. Sunder, J. Med. Chem. 16, 342 (1973).

6 M. M. Abdel-Monem and P. S. Portoghese, J. Med. Chem. 15, 208 (1972).

7 T. A. Montzka, J. D. Matiskella and R. A. Partyka, Tetrahedron Lett. 1974, 1325.

8 T. B. Windholz and D. B. R. Johnston, Tetrahedron Lett. 1967, 2555.

9 M. Bergmann and L. Zervas, Chem. Ber. 65, 1192 (1932).

10 A. M. Felix, E. P. Heimer, T. J. Lambros, C. Tzougraki and J. Meienhofer, J. Org. Chem. 43, 4194 (1978).

11 D. Ben-Ishai and A. Berger, J. Org. Chem. 17, 1564 (1952).

12 A. R. Mitchell and R. B. Merrifield, J. Org. Chem. 41, 2015 (1976).

13 S. Matsuura, C. H. Niu and J. S. Cohen, J. Chem. Soc. Chem. Commun. 1976, 451.

14 H. Yajima, N. Fujii, H. Ogawa and H. Kawatani, J. Chem. Soc. Chem. Commun. 1974, 107.

15 R. S. Lott, V.S. Chauhan and C. H. Stammer, J. Chem. Soc. Chem. Commun. 1979, 495.

16 M. E. Jung and M. A. Lyster, J. Chem. Soc. Chem. Commun. 1978, 315.

17 V. G. Mairanovsky, Angew. Chem. Int. Ed. Engl. 15, 281 (1976).

18 J. Pless and W. Bauer, Angew. Chem. Int. Ed. Engl. 12, 147 (1973).

19 W. Wiegrebe, U. Krüger, H. Reinhart and L.Faber, Arch. Pharm. (Weinheim) 301, 50 (1968).

20 W. Wiegrebe, H. Reinhart and J. Fricke, Pharm. Acta Helv. 48, 420 (1973).

21 Org. Synth. Coll. Vol. 3, 410 (1955). 